\title{
The Pacific Megagash: A future plate boundary?
}

\author{
J.-P. Montagner ${ }^{1}$, D.L. Anderson ${ }^{2}$
}

January 24, 2015

${ }^{1}$ Laboratoire de Sismologie, UMR7154, Institut de Physique du Globe, 1 rue Jussieu, 75238 Paris cedex 05, France. E-mail: jpm@ipgp.fr

${ }^{2}$ Seismological Laboratory, California Institute of Technology, Pasadena, CA91125, U.S.A. (submitted on 23 September 2014)

\begin{abstract}
Seismic anisotropy is an efficient way to investigate the deformation field within the upper mantle. In the framework of rigid tectonic plates, we make use of recent tomographic models of azimuthal anisotropy for deriving the best rotation pole of the Pacific plate in the uppermost $200 \mathrm{~km}$ of the mantle. It is found to be in good agreement with current plate motion (NUVEL1, HS3 and NNR). However, when dividing the Pacific plate into two subplates, separated by the megagash, an E-W low velocity and anisotropy band extending across the Pacific plate from Samoa-Tonga to the Easter- Juan Fernandez Islands, the rotation pole of northern Pacific is still in agreement with current plate motion but not the rotation pole of the southern part of the Pacific, far away from the "classical" rotation pole of the Pacific plate. This result suggests a differential motion between
\end{abstract}


North and South Pacific, and an ongoing reorganisation of plates in the Pacific ocean. The megagash might be a future plate boundary between the North and the South Pacific plates, associated with the intense volcanism along this band.

\section{Introduction}

A wide band of anomalous bathymetry and magmatism extends across the Pacific plate from the Samoa-Tonga elbow to the Easter Island- Juan Fernandez microplate on the East Pacific plate. On the east, this band continues across the Nazca plate, the Challenger fracture zone and the Juan Fernandez volcanic chain to the south end of the volcanic gap in Chile. The distribution of volcanoes, earthquakes and continental geology in Chile suggests an inland interaction with this zone. In the middle of this band, the Easter hotline exhibits bilateral age progressions. It forms the southern boundary of the Easter microplate and lies along the Easter fracture zone. It does not have a distinct swell and it is part of the Tuamotu-Pitcairn-Easter-Sala-y-Gomez tectonic trend, which is parallel to the Samoa-Cook-Austral-Foundation-Juan Fernandez trend. Both trends are apparently related to a change in Pacific plate stress or motion (Clouard and Gerbault, 2008) and the formation of the Easter and Juan Fernandez microplates (Mammerickx, 1992). These trends extend from the Tonga trench to the Chile trench, crossing the East Pacific Rise near the microplates. On the west, this band extends up to the western Pacific along the complex plate boundary between the Pacific plate and the Indo-Australian plate.

This elongated zone, longer than $90^{\circ}$ is much larger than the Central Pacific superswell (McNutt, 1988), since it extends over one-quarter of the Earth's circumference and we referred to it as the Central Pacific megagash (or quadrisect). Smoot and Leybourne (2001) also mentioned this anomalous lineament in Central Pacific, and named it mega- 
trend. Most of the volcanism and seismicity of the Pacific plate are in this complex zone. The megagash can be described as a tectonic and volcanic feature with five or six regions volcanically active at any given time, or as a fortuitous alignment of five or six independent hotspots. Clearly, the number of hotspots, and plumes proposed in this region is hypothesis dependent and might be alternatively the shallow result of passive upwellings or of deep active upwellings. The geodynamic role of the megagash is not yet clearly understood.

This band is also reflected on seismic velocities and anisotropy. The seismic upper mantle structure beneath the Pacific ocean was investigated for many years. The Pacific plate is considered as the simplest example of oceanic plate with thickening of the plate with increasing age $\mathrm{T}$ of the sea floor (Leeds, 1975). However, some deviations with respect to the $\sqrt{T}$ were found in the bathymetry and seismic velocities of central Pacific (Superswell of McNutt and Fisher, 1987) and in the oldest parts of the Pacific plate (Parsons and Sclater, 1977; Ritzwoller et al., 2004; Maggi et al., 2006; Debayle and Ricard, 2013).

Seismic tomography of velocity and anisotropy enables to investigate the origin at depth of this anomalous wide band, the megagash. In isotropic seismic tomography, all global or regional models of Pacific show that the slow S-wave velocities visible below the East-Pacific Rise extend westwards to Polynesia, suggesting close relationship between EPR and Pacific Superswell. Seismic heterogeneities are usually interpreted in terms of heterogeneous temperature field, but a significant part of lateral variations are also induced by seismic anisotropy of upper mantle minerals (Forsyth, 1975; Montagner, 1985; 2002; Smith et al., 2004, Maggi et al., 2006, ...). Due to the predominance of the strongly anisotropic olivine in the upper mantle, and the existence of the lattice preferred orienta- 
tion of olivine induced by convective flow (Nicolas et al., 1973; Christensen and Lundquist, 1982), the fast axis of olivine aligns in the flow direction mapping convection in the upper mantle (Anderson and Regan, 1983). To first order, the azimuthal anisotropy below the Pacific reflects the plate motion as a whole (Montagner, 1994), particularly at the base of the lithosphere and in the asthenosphere in the depth range $80-200 \mathrm{~km}$. It is in good agreement with current kinematic models of plate motions, suggesting a good coupling between lithospheric plate and asthenosphere.

Since seismic anisotropy is a good marker of deformation within lithosphere and asthenosphere, new insight on convection processes, its relationship with plate tectonics and the 3D-structure of the megagash can be gained by taking seismic anisotropy into account in the inversion procedure.

\section{Anisotropy Tomography below the Pacific Ocean}

When developing an anisotropic tomographic technique, two kinds of observables can be measured, the radial anisotropy $\xi$ expressing the difference in S-wave velocity propagating vertically or horizontally (Mitchell and Yu, 1980; Montagner, 1985), and the azimuthal anisotropy of S-wave velocity derived from Rayleigh waves (Forsyth, 1975). Radial anisotropy, already included in PREM (Dziewonski and Anderson, 1981) down to 220km, was derived on a global scale (Nataf et al., 1986; Montagner and Tanimoto, 1991; Ekström and Dziewonski, 1998; ...) to suppress the discrepancy between Rayleigh and Love wave dispersions. Its lateral variations are found as large as isotropic ones down to $200 \mathrm{~km}$ depth, particularly in the center of the Pacific plate (Montagner and Tanimoto, 1991; Ekström and Dziewonski, 1998). Azimuthal anisotropy models through the parameters $G$ and azimuth $\Psi_{G}$ (azimuthal variation of $V_{S V}$ seismic velocity) were first derived 
on a global scale by using initially GDSN data (Montagner and Tanimoto, 1991), and, the dataset was complemented by the large collection of surface wave dispersion data obtained from the FDSN (Federation of Digital Seismic networks) by Ekström et al. (1997) and Ekström (2011). New anisotropic models of the upper mantle have been obtained on a global scale from surface waves (Montagner, 2002, Trampert and Woodhouse, 2003; Debayle et al., 2005) and more recently by Debayle and Ricard (2013), Schaeffer and Lebedev (2013) and Burgos et al. (2014). At the Pacific plate-scale, it has been shown that the azimuthal anisotropy is correlated with present and past plate motion in the depth range 60-250km (Nishimura and Forsyth, 1989), which was confirmed by all recent tomographic models.

Superimposed on the large scale radial and azimuthal anisotropies within and below the lithosphere, the anisotropic tomographic model displays smaller scale $(<1,000 \mathrm{~km})$ lateral variations of these parameters. Two kinds of channels with low anisotropy (hereafter referred as LAC) are observed down to depth of 200km (Montagner, 2002). A first one only observed on azimuthal anisotropy extends from south-west Pacific up to south-east Hawaii and might be the signature of the birth of convective small-scale instabilities in the asthenosphere when the lithosphere cools (Richter and Parsons, 1975; Crambes and Davaille, 2004). Another recently discovered manifestation of small-scale convection is the finger-like structures, present at the base of lithosphere (French et al, 2013). A second kind of low anisotropy channel is observed at the same time on radial and azimuthal anisotropy (Figure 1). It extends between Solomon islands, Samoa-Tonga elbow up to Easter island and across the Nazca plate. Therefore it is at the same location as the wide band of anomalous bathymetry and magmatism that we previously named Pacific megagash. 


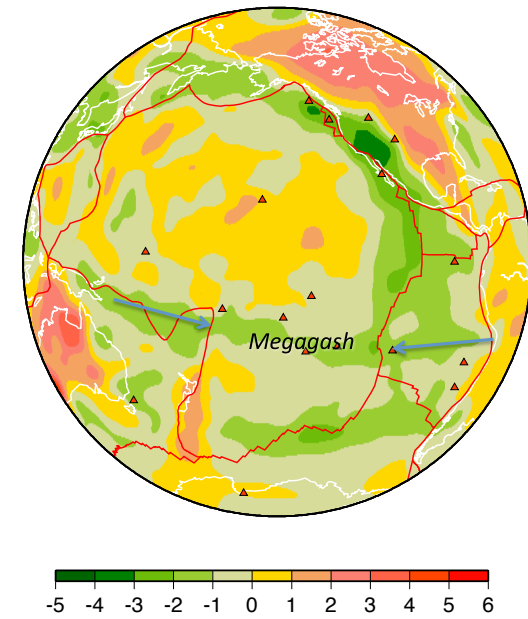

a) Radial anisotropy

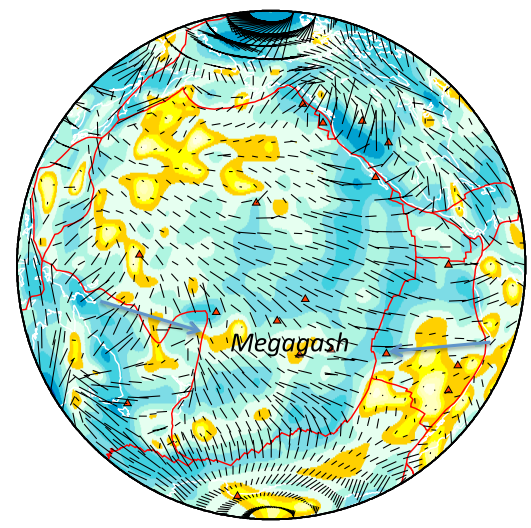

$0.0 \quad 0.10 .2 \quad 0.3 \quad 0.4 \quad 0.6 \quad 1.0 \quad 1.5 \quad 2.0 \quad 3.0 \quad 5.0$

b) Azimuthal anisotropy

Figure 1: Seismic anisotropy parameters, residual radial anisotropy $\delta \xi$ and azimuthal anisotropy of amplitude $G$ and azimuth $\Psi_{G}$ at a depth of $140 \mathrm{~km}$ obtained from the tomographic model of Montagner (2002). The east-west low radial anisotropy $\delta \xi$ (1a) (in green) and less prominent azimuthal anisotropy (1b) bands (in yellow and light blue), named megagash and crossing the Pacific plate, are perfectly visible. $\delta \xi$ is calculated with respect to the reference 1D-model ACY400 (Montagner and Anderson, 1989). Red triangles are the main hotspots with significant magmatism.

We explore in the following sections how the azimuthal anisotropy in the uppermost $300 \mathrm{~km}$ mantle is related to plate motion and we show that the seismic signature of the megagash might possibly be the surface expression of an incipient plate boundary between the North and South Pacific. 


\section{Kinematic rigid motion from seismic anisotropy}

Plate tectonics relies on the assumption that a plate is rigid and that its surface motion can be described by the Euler formula:

$$
\mathrm{V}(\mathrm{M})=\Omega \times \mathrm{OM}
$$

where $\mathbf{V}(\mathbf{M})$ is the velocity of the plate at point $\mathbf{M}(\theta, \phi)$ (where $\theta$ is its colatitude, $\phi$ its longitude), $\mathbf{O}$ the center of the Earth, and $\boldsymbol{\Omega}$ is the rotation pole vector. We can easily calculate, from this equation, the azimuth $\Psi_{\Omega}$ measured clockwise from north at point M. Plate motion induces a strong deformation at the base of the lithosphere, which will induce azimuthal anisotropy (Christensen and Lundquist, 1982), primarily parallel to the plate velocity (see for example Montagner, 1994).

Conversely, the distribution of observed azimuths $\Psi^{\text {obs }}(\theta, \phi)$ for a given parameter such as azimuthal anisotropy enables us to gain insight about rotation poles of tectonic plates. By using this distribution of available $\Psi_{j}^{o b s}$ in a finite number of points $M_{j}$ within the plate, it is possible to retrieve in a least squares sense the best Eulerian rotation pole $\mathbf{P}_{\boldsymbol{\Omega}}\left(\theta_{\Omega}, \phi_{\Omega}\right)$ (surface point of $\boldsymbol{\Omega}$ ). The simplest way to proceed to retrieve $\boldsymbol{\Omega}$ consists in systematically exploring the space of rotation poles characterised by 2 angles $\left(\theta_{\Omega}, \phi_{\Omega}\right)$ with $0^{0}<\phi_{\Omega}<360^{0}$ and $0^{0}<\theta_{\Omega}<180^{\circ}$. It must be noted that the main limitation of this approach is that we do not have access to the amplitude of $\boldsymbol{\Omega}$, but only to angles. Then the corresponding azimuth $\Psi_{\Omega}\left(\theta_{j}, \phi_{j}\right)$ at point $\mathrm{M}$ is calculated, and the associated cost function $\chi^{2}\left(\theta_{\Omega}, \phi_{\Omega}\right)$ at all points $\mathrm{M}$ in the Pacific plate. The cost function is expressed as follows:

$$
\chi^{2}\left(\theta_{\Omega}, \phi_{\Omega}\right)=\frac{\int_{S_{P L}}\left(1-\cos \left(2 \Psi_{\Omega}(M)-2 \Psi_{o b s}(M)\right)\right) \alpha_{o b s}{ }^{-2} V_{\Omega}{ }^{-2} \sin \theta d \theta d \phi}{\int_{S_{P L}} \alpha_{o b s j}{ }^{-2} V_{\Omega}^{-2} \sin \theta d \theta d \phi}
$$

where $S_{P L}$ is the surface of the plate, and $M(\theta, \phi)$ a point belonging to the plate. The 
difference in azimuths $2\left(\Psi_{\Omega}(M)-\Psi_{o b s}(M)\right)$ is weighted by the amplitude of plate velocity $V_{\Omega}(M)$ and by the amplitude of the observed azimuthal anisotropy $\alpha_{\text {obs }}(M)$ at different depths in order to give more weight to points where plate velocity is large and anisotropy is well above noise level. The integral of the cost function, for a constant spacing in longitude and latitude can be discretized in the following way:

$$
\chi^{2}\left(\theta_{\Omega}, \phi_{\Omega}\right)=\frac{\sum_{j=1}^{n_{P L}}\left(1 .-\cos \left(2 \Psi_{\Omega_{j}}-2 \Psi_{o b s_{j}}\right)\right) \alpha_{o b s j}^{-2} V_{\Omega}^{-2}{ }_{j} \sin \theta_{j}}{\sum_{j=1}^{n_{P L}} \alpha_{o b s j}^{-2} V_{\Omega}^{-2}{ }_{j} \sin \theta_{j}}
$$

where $j$ refers to the selected points $M_{j}$ in the Pacific plate and $n_{P L}$ is the number of points in the plate. The $2 \mathrm{D}$ function $\chi^{2}\left(\theta_{\Omega}, \phi_{\Omega}\right)$ also provides the quality of the determination of $\Omega\left(\theta_{\Omega}, \phi_{\Omega}\right)$ through its skewness and can be related to the probability distribution of the rotation pole $\mathbf{P}_{\boldsymbol{\Omega}}$. In the Bayesian statistical approach for Gaussian normal distribution (see for example Tarantola and Valette, 1982), the cost function $\chi^{2}$ is readily related to the Gaussian density probability function $\rho=\rho_{0} e^{\left(-\chi^{2} / 2\right)}$. The half-widths of the cost distribution (in latitude and longitude) provides a first estimate of the uncertainties on the rotation pole.

This equation involving $\Psi_{o b s_{j}}$ can be applied to the distributions of azimuth $\Psi_{\alpha}$ obtained from azimuthal anisotropy tomographic models at different depths. The cost function will be minimum for the Eulerian pole of the layer. We make the underlying assumption that the azimuthal anisotropy of surface waves in the upper mantle reflects the maximum deformation direction, which is also close or aligned with the plate velocity (Christensen and Lundquist, 1982). It must be pointed out that, since only $2-\Psi$ terms of azimuthal anisotropy can be derived, the direction is only found at $\pm \pi\left( \pm 180^{\circ}\right)$. In other words, the intrinsic uncertainty of $\pi$ in the orientation of the azimuthal anisotropy, implies that the pole and its antipode are both minimizing the cost function, so 2 solutions are found for the rotation pole. For sake of plotting simplicity, the cost function 
$\chi$ is normalised between 0 and 1 , as a function $\widehat{\chi}$, such that its maximum (equal to 1 ) corresponds to the minimum $\chi_{\min }$ of $\chi$.

$$
\widehat{\chi}^{2}\left(\theta_{\Omega}, \phi_{\Omega}\right)=\frac{\chi_{\max }^{2}-\chi^{2}\left(\theta_{\Omega}, \phi_{\Omega}\right)}{\chi_{\max }^{2}-\chi_{\min }^{2}}
$$

The observations on G-parameter expressing the azimuthal anisotropy of $S V$-wave are now used. Different models have been considered but only the results for two of them (Montagner, 2002; Burgos et al., 2014) are presented in this paper. The azimuthal anisotropy retrieved from Rayleigh waves, is determined in the depth range between $50 \mathrm{~km}$ down to $400 \mathrm{~km}$, but we will limit the comparison in the depth range $50-250 \mathrm{~km}$.

\subsection{Average uppermost mantle anisotropy- SKS proxy}

By integrating the parameters $G, \Psi_{G}$ with depth, it is possible to calculate the equivalent synthetic S-wave splitting parameters (delay time and orientation) for vertically propagating S-wave, providing an average of seismic anisotropy in the upper mantle. These parameters are like a proxy of SKS-waves (for vertical propagation) and they make possible quantitative comparisons between SKS-splitting data and surface wave derived azimuthal anisotropy (Montagner et al., 2000). This proxy for SKS anisotropy has been used so far for continents where SKS data are available (Marone and Romanowicz, 2007; Wuestefeld et al., 2009; Yuan and Romanowicz, 2010).

The result of the application of equation (1) to synthetic S-wave splitting is shown in figure $\mathbf{2 a}$, where the maximum of $\widehat{\chi}$ is plotted as a pink star. Two kinematic plate models were considered, HS3-Nuvel1A (Gripp and Gordon, 2002) and NNR-Nuvel1 (Argus and Gordon, 1991). Both corresponding rotation poles are actually very close for the Pacific plate and are also plotted as a blue triangle for HS3 and a blue square for NNR. The 
(a) Pacific Plate as a whole

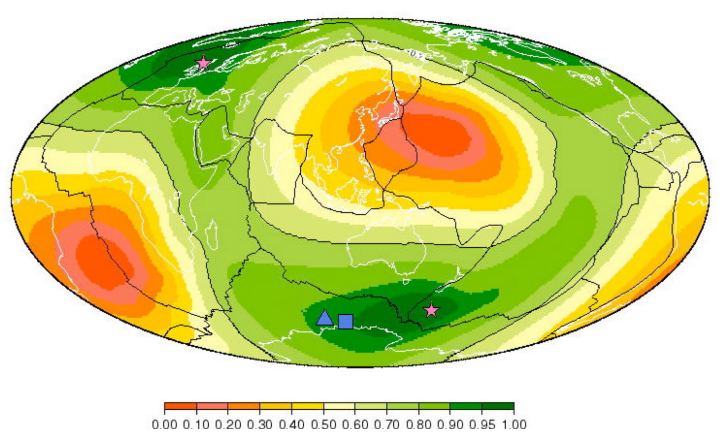

(b) North Pacific Plate

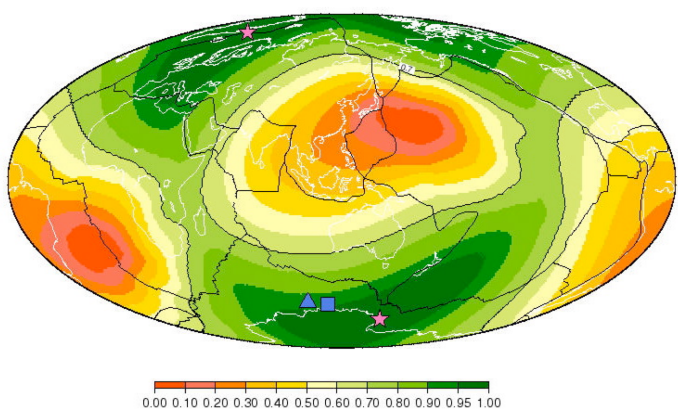

(c) South Pacific Plate

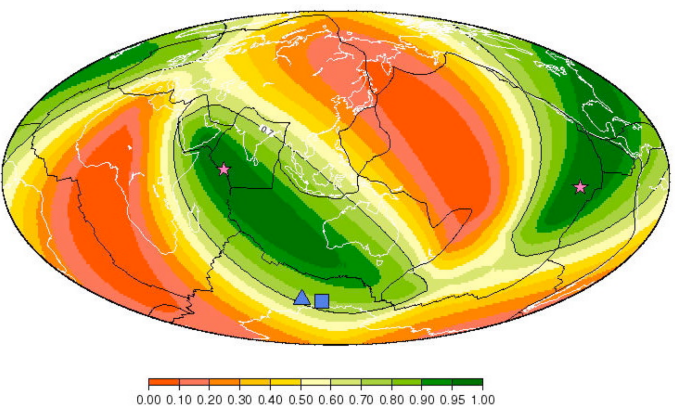

Figure 2: Normalized cost function $\widehat{\chi}\left(\theta_{\Omega}, \phi_{\Omega}\right)$ derived from the distribution of azimuthal anisotropy (Montagner, 2002) within the Pacific plate integrated over the whole depth range 50-250km, giving the equivalent SKS azimuth. (a) the whole Pacific plate. (b) North Pacific plate (north of megagash). (c) South Pacific plate (south of megagash). The pink star corresponds to the maximum probability of the computed rotation derived from azimuthal anisotropy data. The blue triangle (resp. square) is associated with the HS3 (resp. NNR) rotation poles. 
minimum of the cost function $\chi^{2}$ (or maximum of $\widehat{\chi}^{2}$ ) is located close to both rotation poles of the Pacific plate for models HS3 and NNR, in particular in latitude where the difference is smaller than $5^{0}$. The probability distribution as inferred from $\widehat{\chi}^{2}$ is pretty wide in longitude indicating a large uncertainty in $\phi_{\Omega}$ but narrower in latitude. Therefore, to first order, it can be considered that average seismic anisotropy is in good agreement with the Pacific plate velocity directions and can provide interesting informations on the rigid behaviour of the upper mantle.

In a second step, the Pacific plate is divided into a large North Pacific plate and a smaller South Pacific plate separated by the east-west low anisotropy band, also named the megagash in the previous section. The results are presented in figures $\mathbf{2} \mathbf{b}$ for the North Pacific plate and figure 2c for the South Pacific plate for the model of Montagner (2002). The same calculations has been done for the more recent model of Burgos et al. (2014) and the results for SKS proxy (not shown in this paper) are quite similar. As expected, the rotation pole for the North plate is still very close to the rotation pole of a unique Pacific plate, and close to the rotation poles HS3 and NNR. But, the result is more surprising for the South Pacific plate for which the rotation pole is very far away from all of them. The cost function for the South Pacific is rather broad, at the same time in longitude and latitude, but the probability to explain the orientation of azimuthal anisotropy in the South Pacific by the current plate motions HS3 or NNR is very low. Therefore, the averaged distribution of azimuthal anisotropy in the South Pacific lithosphere and asthenosphere is reflecting a difference in the rigid motion with respect to North Pacific. 

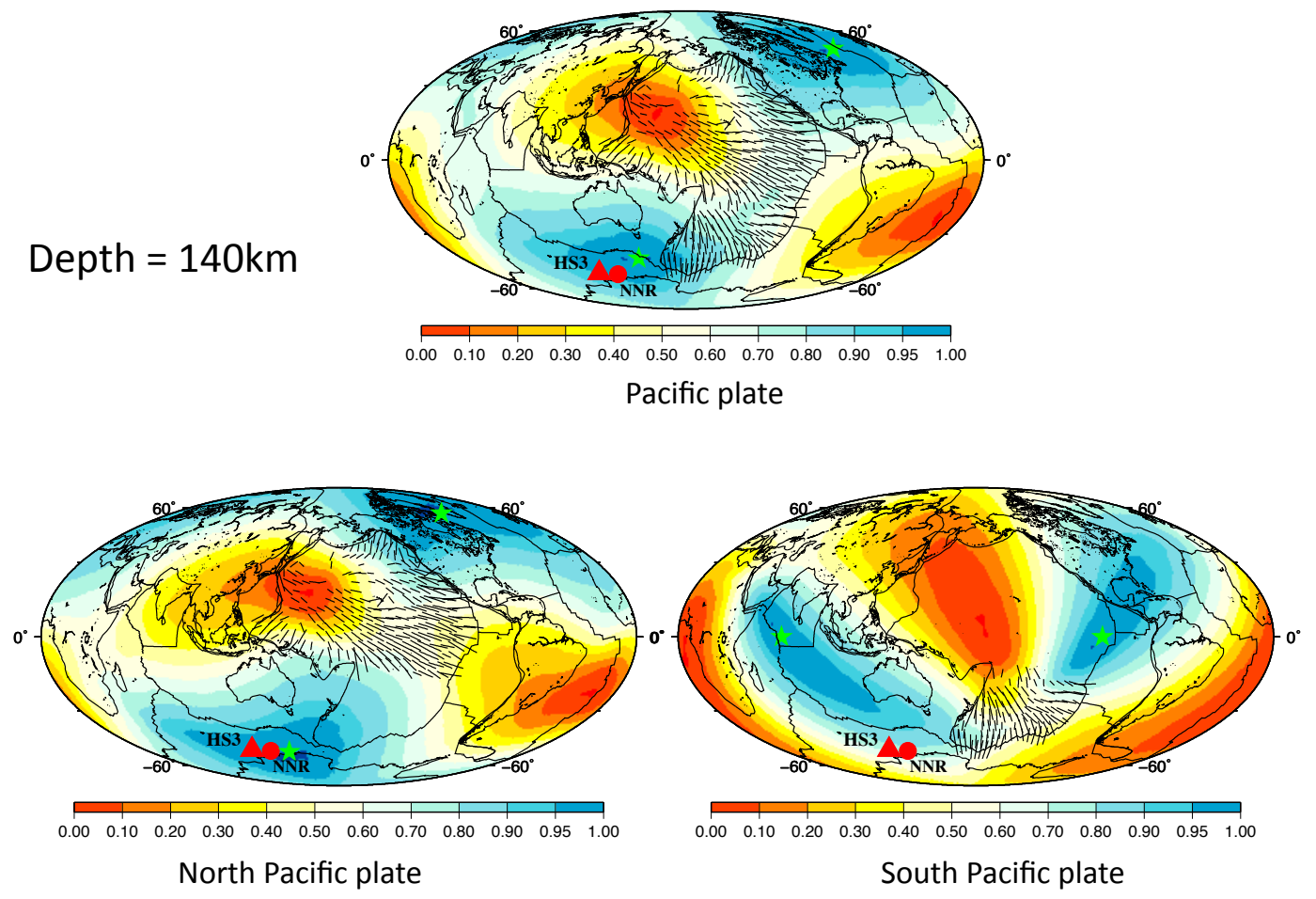

Figure 3: Anisotropy derived poles of rotation for Pacific plate as a whole (top) and the North and South Pacific plates (bottom) obtained from the tomographic model of Montagner (2002) at a depth of 140km. The distribution of azimuthal anisotropy above the error bar at this depth is also plotted.

\subsection{Anisotropy at different depths}

In a third step, we explore the variation of the rotation poles at different depths for North and South Pacific plates. We show in (figures 3) the rotation poles obtained with the model of Montagner (2002) at a depth of $140 \mathrm{~km}$. As previously for the synthetic S-wave splitting map, a significant difference is found between the North Pacific and South Pacific rotation poles. Only the azimuthal anisotropy parameters $G, \Psi_{G}$ smaller than the error bars are taken into account in the calculation of the cost function. The selected points are displayed on the different maps. 
The rotation poles obtained at different depths are shown in Figure 4. For the North Pacific plate, a very good correlation between azimuth $\Psi_{G}$ of anisotropy and plate motion direction is seen below depths of $120 \mathrm{~km}$ down to $180 \mathrm{~km}$. It must be noted that at depths of $50,70 \mathrm{~km}$, the distribution of rotation poles is very broad and does not display well defined maxima. A difference larger than 45 degrees between observed anisotropy pole and plate motion pole is observed. This discrepancy can be interpreted as an indication either of frozen-in (fossil) anisotropy, or of a different orientation mechanism in the upper lithosphere. The correlation between current plate motion and seismic anisotropy derived poles is only valid below $120 \mathrm{~km}$ down to $180 \mathrm{~km}$, where the influence of frozen-in anisotropy is negligible.

The South Pacific rotation poles are significantly different from the North Pacific ones. The discrepancy between North and South Pacific is the largest in the depth range 120$180 \mathrm{~km}$, but below 200km, the rotation poles of North Pacific and South Pacific are more difficult to distinguish, indicating that the coupling between plate tectonics and deep structure tends to vanish below 200km depth (Anderson, 2011).

We also consider other 3D tomographic models of azimuthal anisotropies. For the sake of illustration, we show in Figures 5 the rotation poles obtained with the recent model of Burgos et al. (2014) at a depth of 125km. Again, a significant difference is found between the North Pacific and South Pacific rotation poles in agreement with the results obtained with the model of Montagner (2002). Even though the Burgos' model was obtained with completely different datasets, different methods, the South Pacific rotation poles in the depth 100-180km are still far away the the North Pacific rotation poles which remain close to the HS3 and NNR rotation poles. 


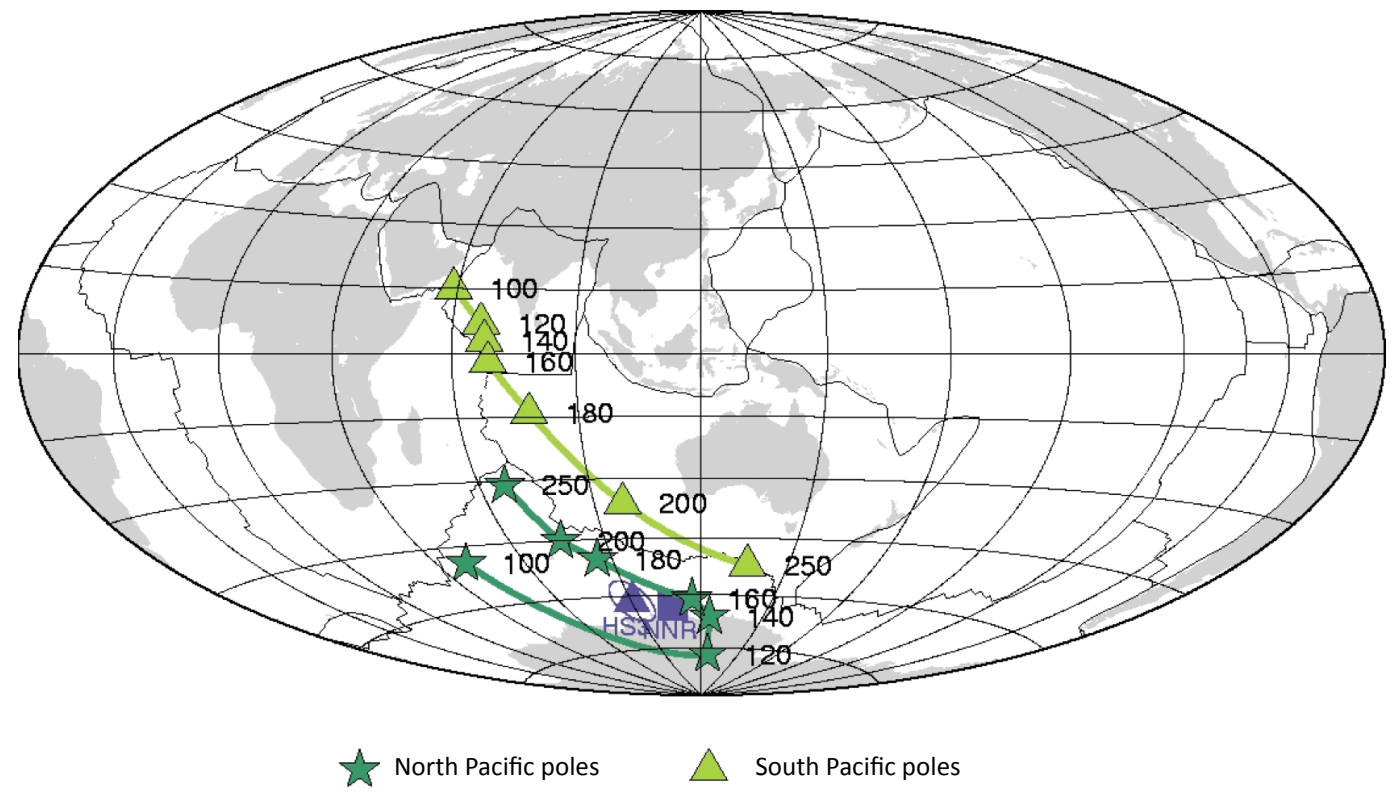

Figure 4: Anisotropy-derived poles of rotation for the North (star) and South (triangle) Pacific plates obtained from the tomographic model of Montagner (2002) at different depths. HS3 and NNR poles are also shown for reference. 


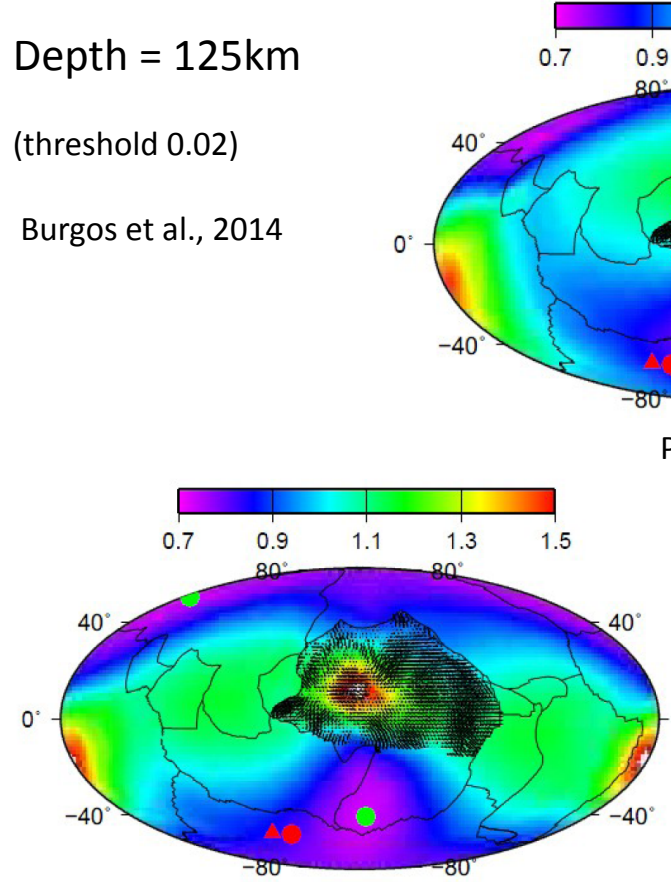

North Pacific plate

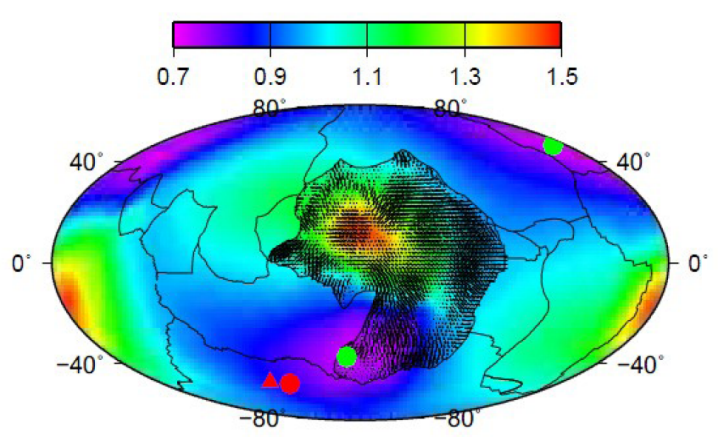

Pacific plate

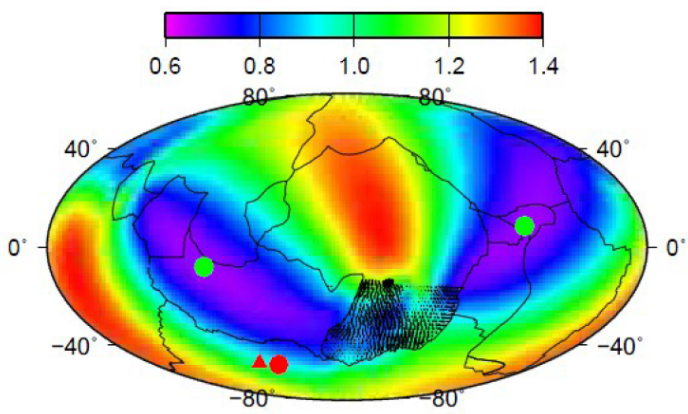

South Pacific plate

Figure 5: Anisotropy-derived poles of rotation for the north and south Pacific plates obtained from the tomographic model of Burgos et al. (2014) at a depth of $125 \mathrm{~km}$. The selected points of the distribution of azimuthal anisotropy at this depth are also plotted. 


\section{Discussion of Geodynamic Implications}

By using the distribution of azimuthal anisotropy in the Pacific plate, our results show first of all, that the coupling in the Pacific plate between lithosphere and asthenosphere in the depth range $120-180 \mathrm{~km}$, is very strong. The existence of well-defined rotation poles down to $180 \mathrm{~km}$ and their correlation with current plate motion means that it reflects a plate tectonics process. Kreemer (2009) followed a similar approach by constraining the absolute plate motions by using SKS shear wave splitting orientations. However, the number of SKS splitting data in the oceanic plates is very limited and measurements are performed only in oceanic islands which are anomalous places (hotspots). The orientation of SKS splitting does not necessarily reflect the underlying large-scale convective motion. In that respect, the use of distributions of azimuthal anisotropy derived from surface waves at different depths, used in this study, is a more efficient way to make comparisons with published plate motion rotation poles.

When separating the Pacific plate into 2 subplates separated by the megagash, we derived the Euler rotation poles for North and South Pacific plates. Whereas the North Pacific pole is close to the usual Pacific Euler pole, the South Pacific pole is significantly different. The different rotation poles of South and North Pacific plates show that there is a differential motion between these 2 subplates (Figure 6 top). The uncertainties on both rotation poles are large, as displayed by the distribution of the cost function. In particular, the South Pacific plate is much smaller than the North Pacific plate. In addition, the distribution of Rayleigh wave paths used for deriving azimuthal anisotropy is poorer than in the north. Consequently, the rotation pole for the South Pacific is not as well resolved as in the North and the very elongated cost function distribution in Figures 2c and 3c, reflects these large uncertainties. However, the South Pacific rotation pole is 
so far away from HS3 and NNR poles that we believe that it is really distinct from these ones.

To find the corresponding rotation pole between North and South Pacific subplates is a tricky and uncertain exercise since we do not have access to the amplitude of the rotations, only their angles. So we did the simplest ad hoc choice $\boldsymbol{\Omega}_{\mathrm{NS}}=\boldsymbol{\Omega}_{\mathrm{N}}-\boldsymbol{\Omega}_{\mathrm{S}}$, corresponding to the same amplitude for North and South subplates. The corresponding orientation of motion is shown at depths of $140 \mathrm{~km}$ and $200 \mathrm{~km}$ (Figure 6 bottom). Just below the lithosphere (140km depth) the eastern part of megagash might be dominated by transform fault and in the western part by opening, whereas, at $200 \mathrm{~km}$ depth, the relative motion is perpendicular to the megagash, suggesting an opening boundary, since it is associated with slow velocities, and a rotational motion between North and South Pacific. Though seismic anisotropy alone does not enable to distinguish between a pole and its antipode, the existence of slow velocities and smaller than average radial anisotropy favours an extensive behaviour for this low anisotropy channel referred to as the Pacific Megagash, also supported by intense intraplate volcanism along this line. It can be interpreted as an incipient plate boundary between the North and South Pacific plates.

At depths larger than $200-250 \mathrm{~km}$, the convective pattern is no longer influenced by plates (Anderson, 2011), the probability distribution is not so well defined, which is due to the decrease of anisotropy amplitude and to the lack of resolution below $200 \mathrm{~km}$ depth.

Tomographic images are only instantaneous views of the present Earth dynamics. The observed seismic anisotropy and its orientation is the intricated result of past deformation (fossil anisotropy) and present-day deformation. But seismic tomography alone cannot provide time constraints necessary to understand the ongoing breakup of the Pacific plate, nor how it might be related to deeper processes. Comparison with other geophysical data 

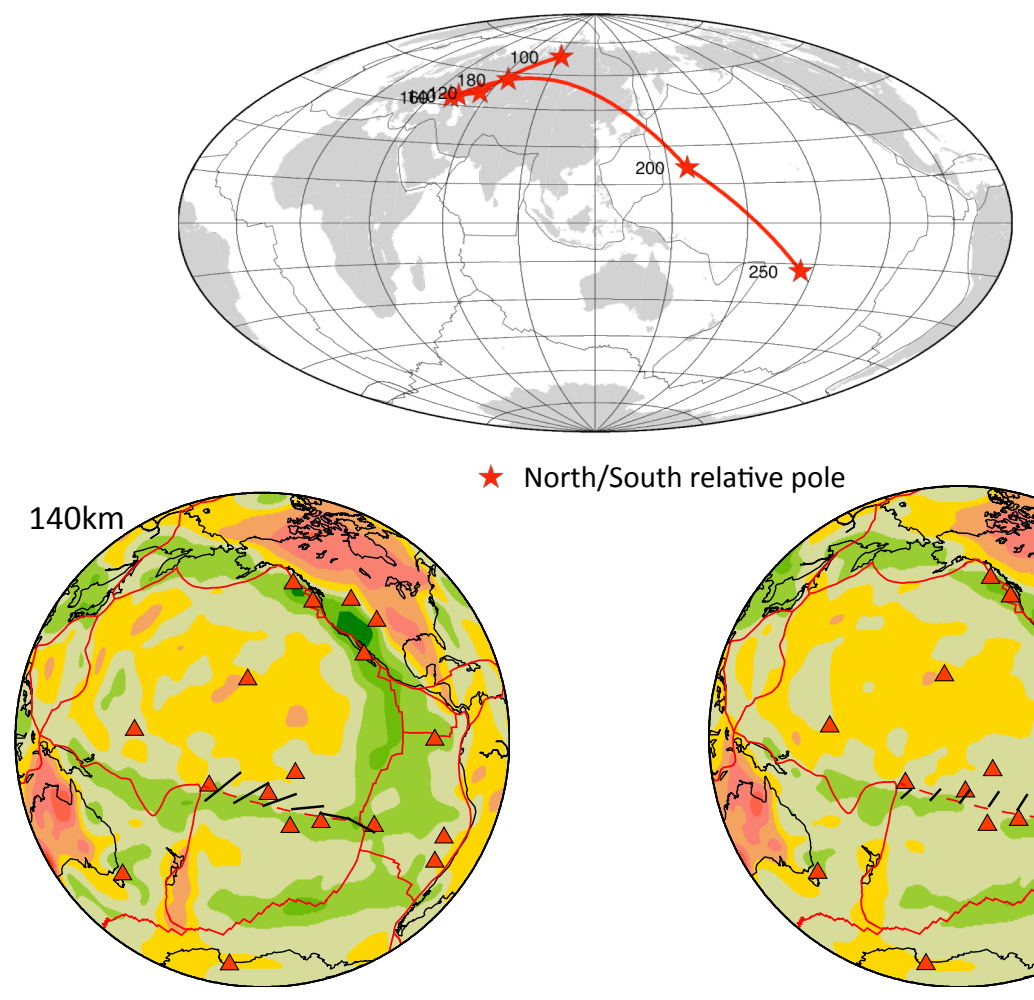

* North/South relative pole

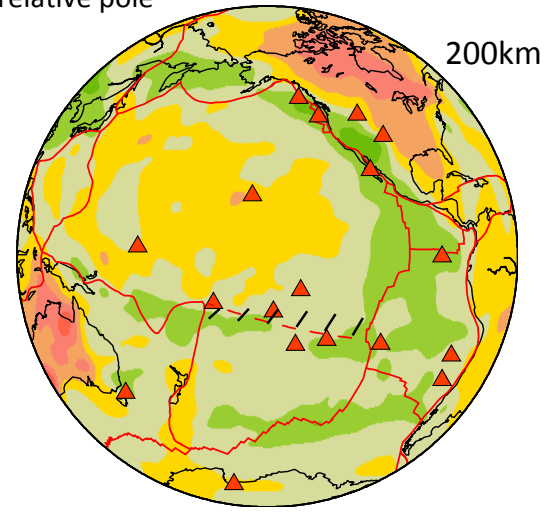

Figure 6: Differential poles of rotation between North and South Pacific plates at different depths. Top: location of differential rotation poles. Bottom left: motion along the megagash at $140 \mathrm{~km}$ depth. Bottom right: same at $200 \mathrm{~km}$ depth. The underlying maps correspond to the radial anisotropy $\delta \xi$ at the same depths from the model of Montagner $(2002)$ 
might be useful. But since there are no islands south of the megagash, there are no geodetic measurements for providing additional constraints on the present-day deformation of the South Pacific plate. The distribution of seafloor ages are different in North and South Pacific and the consequences on the deep convective flow have to be investigated.

The opening of the megagash coupled with slow velocity anomalies in the asthenosphere is a likely explanation for the existence of many hotspots along this band and anomalous intraplate volcanism in south central Pacific. It might preclude a future reorganization of plate boundaries within the Pacific ocean. Considering the geometry of the Pacific plate and its kinematics, Clouard and Gerbault (2008) computed 2D numerical models indicating a shear band between Samoa islands and Easter-Juan Fernandez islands, which leads to the same conclusion. So this process of shearing and breaking present in central Pacific is not necessarily related to a deep plume origin, but might induce the present day intraplate volcanism. It might an excellent example of top-down tectonics (Anderson, 2001).

However, alternative valid explanations can be proposed. So far, we cannot exclude the existence of a deep superplume or several plumes explaining the concentration of hot upwellings in the megagash. These deep upwellings might also contribute to weaken the lithosphere and to the break-up of the Pacific plate. Another important process at play below the Pacific lithosphere is small-scale or secondary convection which takes place at the base of the lithosphere, either in terms of Richter rolls, or viscous fingering. Some of these features have been detected at long wavelengths as low velocity or anisotropy channels (Montagner, 2002; Weeraratne et al., 2007; Harmon et al., 2011; French et al., 2013). How these underlying secondary convective processes might participate to the breakup of rigid plates is still an open question? 
In conclusion, seismic anisotropy seems to imply differential motion between the northern and southern Pacific across the megagash region, and provides good evidence for a future breakup of the Pacific plate along the megagash. But the detailed scenario of the separation between the South and the North Pacific plates is not yet written.

\section{Acknowledgments.}

This work benefited of constructive discussions with Mike Gurnis, Valerie Clouard, Muriel Gerbault at the beginning of the work, of Gael Burgos, and internships of several students, Estelle van Hauwaert, Nawal de Freslon. I also would like to thank Barbara Romanowicz and the second unknown reviewer for their critical and constructive reviews.

\section{References}

Anderson D.L., 1989. The theory of the Earth, Blackwell Scientific Publications, Oxford, 366pp.

Anderson, D.L., 2001. Top-down tectonics? Science, 293, 5537, 2016-2018.

Anderson, D. L, 2011. Hawaii, boundary layers and ambient mantlegeophysical constraints, J. Petr., 52, 1547-1577; doi:10.1093/petrology/egq068.

Anderson D.L., and J. Regan, 1983. Upper mantle anisotropy and the oceanic lithosphere, Geophys. Res. Lett., 10, 841-844.

Burgos, G, J.P. Montagner, E. Beucler, Y. Capdeville, A. Mocquet, and M. Drilleau, 2014. Oceanic lithosphere-asthenosphere boundary from surface wave dispersion data, $J$. Geophys. Res., 119, 1079-1093,

Christensen N.I., and S. Lundquist, 1982. Pyroxene orientation within the upper 
mantle, Bull. Geol. Soc. Am., 93, 279-288.

Clouard V., and M. Gerbault, Muriel, 2008. Break-up spots: Could the Pacific open as a consequence of plate kinematics?, Earth Planet. Sci. Lett., 265, 1, 195-208, 2008.

Crambes C., A. Davaille, A. Lee and J.M. Lees, JM, 2003. Small-scale convection confined under the lithosphere Phys. Earth Planet. Inter..

Debayle E., B.L.N. Kennett, and K. Priestley, 2005. Global azimuthal anisotropy and the unique plate-motion deformation of Australia, Nature, 433, 509-512.

Debayle E., and Y. Ricard, 2012. A Global Shear velocity model of the upper mantle from fundamental and higher Rayleigh mode measurements, J. Geophys. Res. 117, B10308, doi:10.1029/2012JB009288.

Debayle, E. and Ricard Y. 2013., Seismic observations of large-scale deformation at the bottom of fast-moving plates Earth Planet. Sci. Lett., 376, 165-177.

DeMets C., R.G. Gordon, D.F. Argus, and S. Stein, 1990. Current plate motions, Geophys. J. Int., 101, 425-478.

Dziewonski A. M., and D.L. Anderson, 1981. Preliminary Reference Earth Model, Phys. Earth Planet. Int., 25, 297-356.

Ekström G. and A.M. Dziewonski, 1998. The unique anisotropy of the Pacific upper mantle, Nature, 394, 168-172.

Ekström G., J. Tromp, and E.W. Larson, 1997. Measurements and global models of surface wave propagation, J. Geophys. Res., 102, 8137-8157.

Forsyth D. W., 1975. The early structural evolution and anisotropy of the oceanic upper mantle, Geophys. J. R. Astron. Soc., 43, 103-162.

French S., V. Lekic, and B. Romanowicz, 2013. Waveform tomography reveals channeled flow at the base of the oceanic asthenosphere, Science, 342, 6155, 227-230. 
Gripp, A.E., and R.G. Gordon, 2002. Young tracks of hotspots and current plate velocities Geophys. J. Int., 150, 321-361.

Harmon, N., D.W. Forsyth, D.S. Weeraratne,Y. Yang, and S.C. Webb, 2011. Mantle heterogeneity and off axis volcanism on young Pacific lithosphere Earth Planet. Sci. Lett., 311, 3, 306-315.

Kreemer, C., 2009. Absolute plate motions constrained by shear wave splitting orientations with implications for hot spot motions and mantle flow, J. Geophys. Res., Solid Earth, 114, B10, 1978-2012.

Leeds, A.R., 1975., Lithospheric thickness in the western Pacific, Phys. Earth Planet. Int., 11, 61-64.

Maggi, A., E. Debayle, K. Priestley and G. Barruol, 2006. Azimuthal anisotropy of the Pacific region, Earth Planet. Sci. Lett., 250, 53-71.

Mammerickx, J., 1992, The Foundation seamounts: tectonic setting of a newly discovered seamount chain in the South Pacific, Earth Planet. Sci. Lett., 113, 293-306.

Marone F., and B. Romanowicz, 2007. The depth distribution of azimuthal anisotropy in the continental upper mantle Nature, 447, 198-201.

McNutt, M., 1998. Superswell Rev. Geophys., 36, 211244.

McNutt, M., D. Caress, J. Reynolds, K.A. Jordahl, and R.A. Duncan, 1997. Failure of plume theory to explain midplate volcanism in the southern Austral Islands, Nature, 389, 479-482.

McNutt, M. and A. Bonneville, 2000. A shallow chemical origin for the Marquesas swell, Geochem. Geophys. Geosyst., 1, 1999GC00028.

Mitchell B. J., and G.-K. Yu, 1980. Surface wave dispersion, regionalized velocity models and anisotropy of the Pacific crust and upper mantle, Geophys. J. R. Astron. 
Soc., 63, 497-514.

Montagner J.-P., 1985. Seismic anisotropy of the Pacific Ocean inferred from longperiod surface wave dispersion, Phys. Earth Planet. Int., 38, 28-50.

Montagner J.-P., 1994. What can seismology tell us about mantle convection? Rev. Geophys., 32,2, 115-137.

Montagner, J.P. 2002. Upper mantle low anisotropy channels below the Pacific plate, Earth Planet. Sci. Lett., 202, 263-274.

Montagner, J.P., and D.L. Anderson, 1989. Constrained reference Earth model, Phys. Earth Planet. Int., 58, 205- 227.

Montagner J.-P., and T. Tanimoto,1991. Global upper mantle tomography of seismic velocities and anisotropies, J. Geophys. Res. 96, 20,337-20,351.

Montagner J.-P., D.-A. Griot, and J. Lavé, 2000. How to relate body wave and surface wave anisotropies?,J. Geophys. Res. 105 19,015-19,027.

Nataf H.-C., I. Nakanishi, and D.L. Anderson, 1986. Measurement of mantle wave velocities and inversion for lateral heterogeneity and anisotropy, III. Inversion, J. Geophys. Res. 91, 7261-7307.

Nicolas A., F. Boudier, and A.M. Boullier, 1973. Mechanisms of flow in naturally and experimentally deformed peridototes, Am. J. Sci., 273, 853-876.

Nishimura C. E., and D.W. Forsyth, 1989. The anisotropic structure of the upper mantle in the Pacific, Geophys. J. 96: 203-229.

Parsons, B. and Sclater, J.G., 1977. An analysis of the variation of ocean floor bathymetry and heat flow with age, J. Geophys. Res., 82, 5, 803-827.

Richter, F.M., and B. Parsons, 1975. On the interaction of two scales of convection in the mantle, J. Geophys. Res., 80, 2529-2541. 
Ritzwoller, M.H., N. Shapiro, and S.J. Zhong, 2004. Cooling history of the Pacific lithosphere, Earth Planet. Sci. Lett., 226, 69-84.

Schaeffer, A.J. and S. Lebedev, 2013. Global shear speed structure of the upper mantle and transition zone, Geophys. J. Int., ggt095.

Smith, D. B, M.H. Ritzwoller, and N.M. Shapiro, 2004. Stratification of anisotropy in the Pacific upper mantle, J. Geophys. Res., 109, B11, 1978-2012.

Smoot N.C., and B.A. Leybourne, 2001. The Central Pacific Megatrend, Int. Geol. Rev., 43, 341-365.

Tanimoto, T., and D.L. Anderson, 1984. Mapping convection in the mantle, Geophys. Res. Lett., 11, 4, 287-290.

Tarantola, A., and B. Valette, 1982. Generalized nonlinear inverse problems solved using least squares criterion. Rev. Geophys. Space Phys., 20, 219232.

Trampert J., and J.H. Woodhouse, 2003. Global anisotropic phase velocity for fundamental mode surface waves between 40 and 150s, Geophys. J. Int. 154, 154-165.

Weeraratne, D.S., D.W. Forsyth, Y. Yang, and S.C. Webb, 2007. Rayleigh wave tomography beneath intraplate volcanic ridges in the South Pacific J. Geophys. Res., Solid Earth, 112, B06303, doi:10.1029/2006JB004403.

Wüstefeld A., G. Bokelmann, G. Barruol, and J.-P. Montagner 2009. Identifying global seismic anisotropy patterns by correlating shear-wave splitting and surface-wave data, Phys. Earth Planet. Int., 176, 198-212.

Yuan H. and B. Romanowicz, 2010. Lithospheric layering in the North American craton, Nature, 466, 7310, 1063-1068. 